\title{
Impacto de la ISO 9001:2008 en la Facultad de Ingeniería de la Universidad Nacional de Chimborazo - Ecuador como parte del sistema de gestión de calidad
}

\section{RESUMEN}

El propósito de este artículo es determinar el impacto que tiene la norma ISO 9001: 2008 en el sistema de gestión de la calidad en la Facultad de Ingeniería en la Universidad Nacional de Chimborazo- Ecuador. Esta investigación es el resultado de un trabajo de campo, en donde se realizó un análisis de la información recolectada, a través de encuestas validadas por medio del alfa de Cronbach. La muestra es de 200 estudiantes, con un nivel de significación del $95 \%$, las mismas fueron aplicadas a los estudiantes de manera aleatoria, los resultados muestran que el impacto es bueno.

Palabras clave: Impacto bueno, gestión de calidad, alfa de Cronbach.

IMPACT OF ISO 9001: 2008 IN THE FACULTY OF ENGINEERING OF THE NATIONAL UNIVERSITY OF CHIMBORAZO - ECUAdOR AS PART OF THE QUALITY MANAGEMENT SYSTEM

\section{ABSTRACT}

The purpose of this article is to determine the impact of the ISO 9001: 2008 standard on the quality management system at the Faculty of Engineering at the National University of Chimborazo, Ecuador. This research is the result of a field work, where an analysis of the information collected was carried out, through surveys validated by Cronbach's alpha. The sample is of 200 students, with a level of significance of $95 \%$, they were applied to the students in a random way, the results show that the impact is good.

Keywords: Good impact, quality management, Cronbach alpha.

\section{INTRODUCCIÓN}

Al interior de las instituciones de educación superior se llevan a cabo procesos de adaptación estratégicos a las nuevas realidades del siglo XXI, Según la Ley Orgánica de Educación Superior en Ecuador (LOES), la Evaluación de la Calidad es el proceso para determinar las condiciones de la institución, carrera o programa académico, mediante la recopilación sistemática de datos cuantitativos y cualitativos que permitan emitir un juicio o diagnóstico, analizando sus componentes, funciones, procesos, a fin de que sus resultados sirvan para reformar y mejorar el programa de estudios, carrera o institución, pese a que la LOES tiene sus propios indicadores no es menos cierto que es imprescindible aplicar la Norma ISO 9001 con mira a que la misma sirva de base para la acreditación y para ser competitiva en el mercado, según la lengua española mercado es: conjunto de consumidores capaces de comprar un producto o servicio.

La norma ISO 9001:2008 es una norma Europea que fue aprobada por el comité europeo de normalización (CEN) el 8 de noviembre de 2008. Es la base del sistema de gestión de la calidad, entendiéndose como gestión de calidad al conjunto de actividades de la función general, de la dirección que determinan la política de la calidad, los objetivos y las responsabilidades y se implanta por medios tales como la planificación, el control, el aseguramiento y la mejora de la calidad en el marco del sistema de la calidad UNE-EN -ISO 8402, por lo antes expuesto toda Empresa e Institución debe disponer de calidad de su producto o servicio, la calidad en Instituciones de nivel superior está dirigida a las funciones de docencia, investigación y extensión, lo que se traduce en calidad de su personal docente, calidad de sus programas , calidad de sus métodos de enseñanza - aprendizaje, calidad en estudiantes, calidad en su infraestructura, todos estos aspectos determinan el funcionamiento de la universidad y la imagen institucional que proyecta a la sociedad en general,

1 Magister en Diseño, Gestión y Evaluación de Proyectos Sociales y Productivos, Doctor en Ingeniería Industrial, Docente Investigador Titular de la Facultad de Ingeniería de la Universidad Nacional de Chimborazo, Riobamba, Ecuador.

Investigador Titular de la Facultad de Ingeniería de la Universidad Nacional de Chimborazo. Riobamba.Ecuador.

E-mail hsalazar@unach.edu.ec

2 Magister en Diseño, Gestión y Evaluación de Proyectos Sociales y Productivos, Doctor en Ingeniería Industrial, Docente Investigador Titular de la Facultad de Ingeniería de la Universidad Nacional de Chimborazo, Riobamba, Ecuador.

E-mail: mcabrera@unach.edu.ec

3 Magíster en Gestión de la Producción, Doctor en Ingeniería Industrial, Docente Investigador Titular de la Facultad de Ingeniería de la Universidad Nacional de Chimborazo, Riobamba, Ecuador. E-mail: pvillacres@unach.edu.ec 
para Wellintong, (1997) indica "que se debe prestar un servicio acorde con las necesidades de los clientes, y no según la presencia o ausencia de competidores" (pág. 60). Para Juran \& gryna, (1993) "la calidad es el conjunto de características de un producto o servicio que satisface a los clientes, y por lo tanto hacen aceptable el producto" (pàg.20) . "La calidad es la habilidad que posee un sistema para operar de manera fiable y sostenida en el tiempo, a un determinado nivel de Desempeño; en una organización de servicios" (Dominguez, 2006, pág. 47). Mientras que para Ishikawa, (1997), un autor reconocido de la gestión de la calidad, proporcionó la siguiente definición respecto a la Calidad Total: "Filosofía, cultura, estrategia o estilo de gerencia de una empresa o Institución según la cual todas las personas en la misma, estudian, practican, participan y fomentan la mejora continua de la calidad" (pág. 8). Para yanèz, (2008), "calidad es el grado en que un conjunto de características cumple con los requisitos (pág. 2).

En este sentido se puede decir que Is Sistemas de Gestión de la Calidad son un conjunto de normas y estándares internacionales que se interrelacionan entre sí para hacer cumplir los requisitos de calidad que una empresa o Institución requiere para satisfacer los requerimientos acordados con sus clientes a través de la ISO 9001:2008.

Todo sistema de gestión de calidad tiene que ser medible mediante técnicas de investigación, para llevar a cabo dicha medición se empleó un instrumento de encuestas de 24 preguntas con escala de Likert, el mismo que fue otorgado por el departamento de calidad de la facultad. Aunque el servicio ofrecido por la entidad sea intangible será necesario determinar la totalidad de parámetros de medida de manera clara y concisa proporcionando las referencias necesarias para alcanzar buenos resultados.

Esta reflexión se convirtió en el punto de partida para esta investigación que tiene como objetivo general determinar el impacto que tiene la norma ISO
9001:2008 en la facultad de Ingeniería de la Universidad Nacional de Chimborazo.

A través de esta investigación se pretende realizar un aporte al sistema educativo en lo referente al campo administrativo, construyendo un instrumento de recolección de datos validado que permita evaluar el Impacto que tiene la norma ISO y a la vez develar los atributos que para el estudiante es importante con el fin de lograr un mejoramiento.

\section{METODOLOGÍA}

El presente estudio es una investigación de tipo descriptiva, en ella se describe el impacto que tiene las ISO sobre el sistema de gestión de calidad que presta la Facultad de Ingeniería de la Universidad Nacional de Chimborazo a sus estudiantes, Para llevar a cabo la investigación, se procedió a solicitar al coordinador el cuestionario (tabla 1), La recopilación de la información, se lo realizó validando el constructo. Los datos se los obtuvo de los involucrados, esto es de los Estudiantes de la Facultad de Ingeniería, cuya población es de 2240.

\section{ANÁLISIS FACTORIAL}

En la Tabla 2, se observa la prueba de Bartlett, la misma que demuestra que el análisis factorial si es procedente, puesto que el sig es < a 0,05. Este análisis es ratificado por la prueba de káiser, cuyo valor $(0,857)$ debe aproximarse a uno.

En la Tabla 3, se puede observar que el modelo arroja 6 componentes, con un $66,918 \%$ de su varianza total explicada.

La Figura 1 de sedimentación muestra el número de componentes de la investigación que se vuelve a ratificar lo antes dicho por la Tabla 3.

En la Tabla 4, presenta la matriz de componentes rotados. Cada una de las preguntas es agrupada en cada uno de los componentes o factores del modelo. 


\section{UNIVERSIDAD NACIONAL DE CHIMBORAZO \\ FACULTAD DE INGENIERÍA \\ ESCUELA DE INGENIERIA INDUSTRIAL}

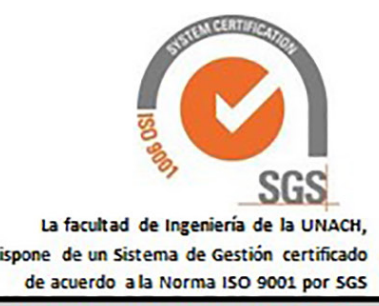

Objetivo: Determinar el grado de satisfacción estudiantil en relación al servicio educativo de la Facultad Ingeniería. Buenos "Días" o "Tardes". Si fuese usted tan amable en responder con toda honestidad del caso, la siguiente encuesta:

1: muy malo 2: malo 3: indiferente 4: bueno 5: muy bueno

Tabla 1. Diseño de encuestas.

\section{Escala de Likert}

$\begin{array}{lllll}1 & 2 & 3 & 4 & 5\end{array}$

La oferta académica de carreras de la facultad de Ingeniería es:

El aprendizaje académico que recibe en su carrera es:

El nivel de formación integral, ética y valores que recibe su carrera es:

Los proyectos de Vinculación con la colectividad ejecutados en su carrera son:

La oportunidad de insertarme laboralmente en el campo ocupacional de mi carrera es

La preparación académica de los docentes de su carrera se considera como:

Los recursos tecnológicos que se emplean en clases son:

La infraestructura con la que cuenta su carrera es:

El apoyo extracurricular (tutorías) que recibe por parte de los Docentes es:

La posibilidad de generar fuentes de empleo a partir del conocimiento académico recibido en la Carrera es:

La atención estudiantil que recibe en las dependencias de Secretaria de la Facultad de Ingeniería es:

La diversidad de Idiomas Extranjeros que usted puede seleccionar en el Centro de Idiomas es:

La preparación académica del Idioma Extranjero que recibe en el Centro de Idiomas es:

El uso e infraestructura del laboratorio de Idiomas es:

La calidad del servicio de la Biblioteca en cuanto a disponibilidad de bibliografía y atención es:

La calidad de servicio de laboratorios en cuanto a disponibilidad de recursos y atención es:

La calidad del servicio de bares en cuanto a alimentación y atención es:

La seguridad en las instalaciones de la facultad de Ingeniería es:

La medida en que se ha implementado becas estudiantiles en la Facultad de Ingeniería es:

La posibilidad de contar con traspasos estudiantiles a otras carreras es:

La implementación aplicada para conseguir la acreditación de carreras de la Facultad de Ingeniería es:

El sistema de Gestión de Calidad en la facultad de Ingeniería bajo la Norma ISO 9001 es:

La metodología de los Docentes al dictar sus respectivas clases es:

Los espacios físicos en donde usted recibe sus clases es:

Fuente: Departamento de calidad de la Facultad de Ingeniería. 
Tabla 2. KMO y prueba de Bartlett.

\begin{tabular}{llr}
\hline \multicolumn{2}{l}{ Medida de adecuación muestral de Kaiser-Meyer-Olkin. } &, 857 \\
\hline \multirow{3}{*}{ Prueba de esfericidad de Bartlett } & Chi-cuadrado aproximado & 1165,573 \\
\cline { 2 - 3 } & gl & 276 \\
\cline { 2 - 3 } & Sig. &, 000
\end{tabular}

Fuente: Elaboración propia.

Tabla 3. Varianza total explicada.

\begin{tabular}{|c|c|c|c|c|c|c|c|c|c|}
\hline \multirow{2}{*}{ Componente } & \multicolumn{3}{|c|}{ Auto valores iniciales } & \multicolumn{3}{|c|}{$\begin{array}{l}\text { Sumas de las saturaciones al } \\
\text { cuadrado de la extracción }\end{array}$} & \multicolumn{3}{|c|}{$\begin{array}{l}\text { Suma de las saturaciones al } \\
\text { cuadrado de la rotación }\end{array}$} \\
\hline & Total & $\begin{array}{c}\% \text { de la } \\
\text { varianza }\end{array}$ & $\begin{array}{c}\% \\
\text { acumulado }\end{array}$ & Total & $\begin{array}{c}\% \text { de la } \\
\text { varianza }\end{array}$ & $\begin{array}{c}\% \\
\text { acumulado }\end{array}$ & Total & $\begin{array}{c}\% \text { de la } \\
\text { varianza }\end{array}$ & $\begin{array}{c}\% \\
\text { acumulado }\end{array}$ \\
\hline 1 & 9,880 & 41,166 & 41,166 & 9,880 & 41,166 & 41,166 & 3,850 & 16,043 & 16,043 \\
\hline 2 & 1,664 & 6,933 & 48,099 & 1,664 & 6,933 & 48,099 & 3,371 & 14,048 & 30,090 \\
\hline 3 & 1,299 & 5,412 & 53,511 & 1,299 & 5,412 & 53,511 & 2,855 & 11,897 & 41,988 \\
\hline 4 & 1,156 & 4,818 & 58,330 & 1,156 & 4,818 & 58,330 & 2,364 & 9,851 & 51,839 \\
\hline 5 & 1,059 & 4,411 & 62,741 & 1,059 & 4,411 & 62,741 & 2,151 & 8,963 & 60,802 \\
\hline 6 & 1,003 & 4,177 & 66,918 & 1,003 & 4,177 & 66,918 & 1,468 & 6,117 & 66,918 \\
\hline 7 & ,911 & 3,795 & 70,713 & & & & & & \\
\hline 8 & 845 & 3,521 & 74,234 & & & & & & \\
\hline 9 & ,777 & 3,236 & 77,470 & & & & & & \\
\hline 10 & 643 & 2,679 & 80,149 & & & & & & \\
\hline 11 &, 596 & 2,483 & 82,632 & & & & & & \\
\hline 12 &, 587 & 2,445 & 85,077 & & & & & & \\
\hline 13 &, 521 & 2,169 & 87,246 & & & & & & \\
\hline 14 & ,475 & 1,979 & 89,225 & & & & & & \\
\hline 15 & ,463 & 1,930 & 91,155 & & & & & & \\
\hline 16 & ,409 & 1,702 & 92,857 & & & & & & \\
\hline 17 & ,318 & 1,324 & 94,181 & & & & & & \\
\hline 18 & 299 & 1,245 & 95,426 & & & & & & \\
\hline 19 & ,254 & 1,060 & 96,486 & & & & & & \\
\hline 20 & ,224 & ,935 & 97,421 & & & & & & \\
\hline 21 & , 184 & ,765 & 98,186 & & & & & & \\
\hline 22 & 168 & 699 & 98,885 & & & & & & \\
\hline 23 & 150 & ,626 & 99,511 & & & & & & \\
\hline 24 & 117 & ,489 & 100,000 & & & & & & \\
\hline
\end{tabular}

Método de extracción: Análisis de Componentes principales.

Fuente. Elaboración propia. 


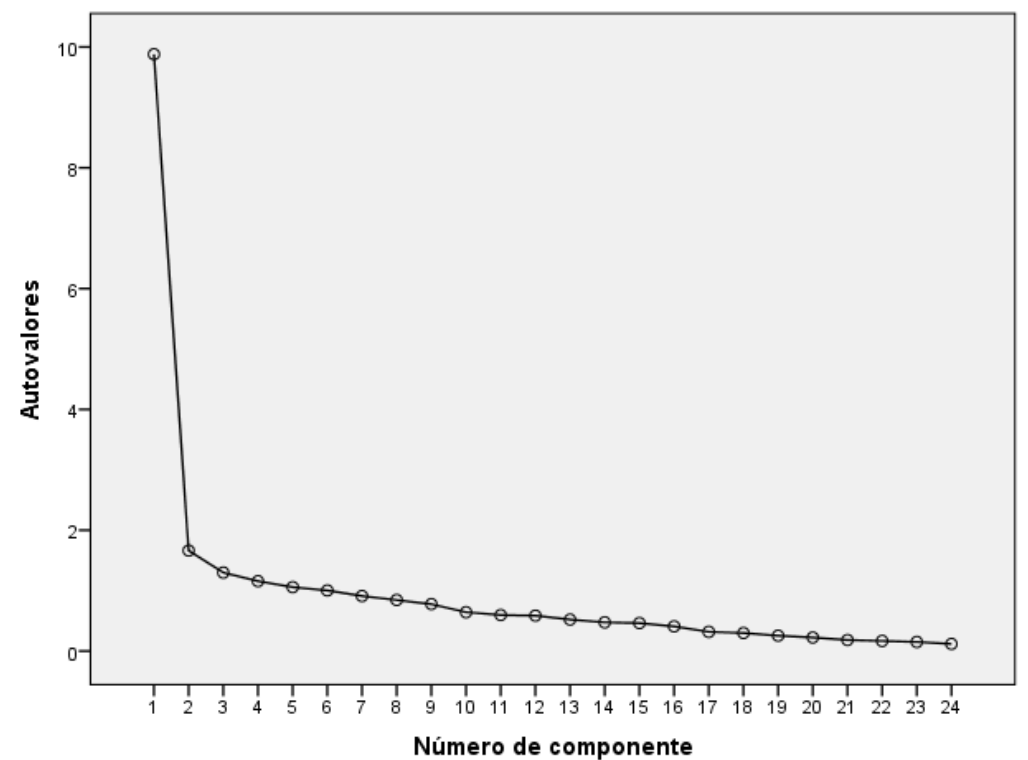

Fuente: Elaboración propia.

Figura 1. Gráfico de sedimentación.

Tabla 4. Matriz de componentes rotadosa.

\begin{tabular}{|c|c|c|c|c|c|c|}
\hline & \multicolumn{6}{|c|}{ Componente } \\
\hline & 1 & 2 & 3 & 4 & 5 & 6 \\
\hline 1. La oferta académica de la carrera de la facultad de Ingeniería es & 040 & ,490 &, 575 & ,223 &,- 080 & 099 \\
\hline 2. El aprendizaje académico que recibe en su carrera es & ,382 & ,178 & ,393 & ,216 & ,425 & 126 \\
\hline 3. El nivel de formación integral ética y valores que recibe su carrera es & ,115 &,- 039 & ,697 & , 145 &, 271 &, 419 \\
\hline 4. Los proyectos de vinculación con la colectividad ejecutados en su carrera es & ,337 & ,116 & 115 & ,432 & ,423 & ,294 \\
\hline 5. La oportunidad de insertarme laboralmente en el campo ocupacional de mi carrera es & , 127 & ,262 & 059 &, 012 & ,169 & ,844 \\
\hline 6. La preparación académica en los docentes de su carrera se considera como &, 012 & ,401 &, 556 &, 252 & ,361 & 056 \\
\hline 7. Los recursos tecnológicos que se emplean en clases son & ,173 & ,378 & ,368 & ,473 & ,278 &,- 191 \\
\hline 8. La infraestructura con la que cuenta su carrera es & ,145 & ,673 & 101 & ,182 & , 182 & , 141 \\
\hline 9. El apoyo extracurricular (tutorías) que recibe por parte del docente es &,- 074 & ,143 & 074 & ,201 & ,742 & , 165 \\
\hline 10. La posibilidad de generar fuentes de empleo a partir del conocimiento académico recibido en su carrera es & ,009 &, 563 & ,093 &, 384 & 383 & 199 \\
\hline 11. La atención estudiantil que recibe en la dependencias de Secretaria de la facultad de Ingeniería es & ,345 & ,103 &, 115 &, 779 & ,238 &,- 101 \\
\hline 12. La diversidad de idiomas extranjeros que ud puede seleccionar en el centro de Idiomas es & ,284 & ,306 & ,359 &, 514 & ,030 & ,264 \\
\hline 13. La preparación académica del idioma extranjero que recibe en el centro de idiomas es &, 598 & ,204 & ,293 & ,191 & ,105 & ,229 \\
\hline 14. El uso y la infraestructura del laboratorio de idiomas es & ,785 & ,183 & , 185 &, 160 & ,115 & ,137 \\
\hline 15. La calidad de servicios en la biblioteca es en cuanto a la disponibilidad de bibliografía y atención es & ,460 & ,216 & 647 &, 138 & ,008 &,- 294 \\
\hline 16. La calidad de servicios de laboratorios en cuanto a la disponibilidad de recursos y atención es & ,343 & ,491 &, 502 & 131 & 091 &,- 107 \\
\hline 17. La calidad de servicios de bares en cuanto alimentación y atención es &, 500 & ,215 & ,207 & ,489 &,- 050 & ,156 \\
\hline 18. La seguridad en las instalaciones de la facultad de Ingeniería es & ,319 & ,701 & 120 & , 155 & ,121 & ,191 \\
\hline 19. La medida que se ha implementado becas estudiantiles en la facultad de Ingeniería es & ,414 &, 510 & ,287 & ,243 &,- 080 & ,119 \\
\hline 20. La posibilidad de contar con traspasos estudiantiles a otras carreras es &, 732 & ,174 & ,007 & ,194 & ,022 &,- 067 \\
\hline 21. La implementación aplicada para seguir la acreditación de carreras de la facultad de Ingeniería es & ,607 & ,209 &, 115 & ,454 & , 146 & , 077 \\
\hline 22. El sistema de Gestión de Calidad en la facultad de Ingeniería bajo la Norma ISO 9001 es & ,430 & 138 & ,499 &,- 057 & 387 &, 068 \\
\hline 23. La metodología de los docentes al dictar sus respectivas clases son &, 503 & ,273 & 177 &,- 045 & ,634 &,- 058 \\
\hline 24. Los espacios físicos en donde usted recibe sus clases es & ,367 & ,699 & 183 &,- 101 & ,241 &,- 020 \\
\hline
\end{tabular}

Método de extracción: Análisis de componentes principales.

Método de rotación: Normalización Varimax con Kaiser.

a. La rotación ha convergido en 14 iteraciones.

Fuente: Elaboración propia. 


\section{Dimensiones del Análisis Factorial}

En la Tabla 5, se encuentran las preguntas del cuestionario agrupadas por factores o dimensiones. En la dimensión de acreditación se agrupa 6 pregun- tas, Para la dimensión de tangibilidad 4 preguntas, para la dimensión de académico 6 , para la dimensión de empatía 4 y para la dimensión de tutorías 2 pregunta.

Tabla 5. Dimensiones.

\begin{tabular}{l}
\hline \multicolumn{1}{c}{ Universidad Nacional de Chimborazo } \\
\hline Dimensiones del cuestionario \\
\hline 1. La preparación académica del idioma extranjero que recibe en el centro de idiomas es \\
2. El uso y la infraestructura del laboratorio de idiomas es \\
3. La calidad de servicios de bares en cuanto alimentación y atención es \\
4. La posibilidad de contar con traspasos estudiantiles a otras carreras es \\
5. La implementación aplicada para seguir la acreditación de carreras de la facultad de Ingeniería es \\
6. El sistema de Gestión de Calidad en la facultad de Ingeniería bajo la Norma ISO 9001 es
\end{tabular}

1. La infraestructura con la que cuenta su carrera es

2. La posibilidad de generar fuentes de empleo a partir del conocimiento académico recibido en su carrera es

Tangibilidad 3. La seguridad en las instalaciones de la facultad de Ingeniería es

4. La medida que se ha implementado becas estudiantiles en la facultad de Ingeniería es

5. Los espacios físicos en donde usted recibe sus clases es

1. La oferta académica de la carrera de la facultad de Ingeniería es

2. El aprendizaje académico que recibe en su carrera es

Académico

3. El nivel de formación integral ética y valores que recibe su carrera es

4. La preparación académica en los docentes de su carrera se considera como

5. La calidad de servicios en la biblioteca es en cuanto a la disponibilidad de bibliografía y atención es

6. La calidad de servicios de laboratorios en cuanto a la disponibilidad de

1. Los proyectos de vinculación con la colectividad ejecutados en su carrera es

Empatía

2. Los recursos tecnológicos que se emplean en clases son

3. La atención estudiantil que recibe en la dependencias de Secretaria de la facultad de Ingeniería es

4. La diversidad de idiomas extranjeros que Ud. puede seleccionar en el centro de Idiomas es

Tutoría

1. El apoyo extracurricular (tutorías) que recibe por parte del docente es

2. La metodología de los docentes al dictar sus respectivas clases son

Fuente. Elaboración propia.

\section{Análisis de confiabilidad}

El análisis de confiabilidad tiene que ver con el alfa de Cronbach, el mismo que tiene que ser mayor a 0,5 . Esto significa que todas las preguntas que pertenecen a la dimensión contribuyen a explicar la misma dentro de la investigación. En este sentido las Tablas (6-7-8-9-10) disponen de un alfa de Cronbach superior a 0,5 .
Estadísticos de fiabilidad

Tabla 6. Acreditación.

\begin{tabular}{cc}
\hline Alfa de Cronbach & $\mathbf{N}^{\circ}$ de elementos \\
\hline, 812 & 6 \\
\hline
\end{tabular}

Fuente. Elaboración propia. 
Estadísticos de fiabilidad

Tabla 7. Tangibilidad.

\begin{tabular}{cc}
\hline Alfa de Cronbach & $\mathbf{N}^{\circ}$ de elementos \\
\hline, 817 & 5 \\
\hline
\end{tabular}

Fuente. Elaboración propia.

Estadísticos de fiabilidad

Tabla 8. Académico.

\begin{tabular}{cc}
\hline Alfa de Cronbach & $\mathbf{N}^{\circ}$ de elementos \\
\hline, 824 & 6 \\
\hline
\end{tabular}

Fuente: Elaboración propia.

Estadísticos de fiabilidad

Tabla 9. Empatía.

\begin{tabular}{cc}
\hline Alfa de Cronbach & $\mathbf{N}^{\circ}$ de elementos \\
\hline, 766 & 4 \\
\hline
\end{tabular}

Fuente: Elaboración propia.

Estadísticos de fiabilidad

Tabla 10. Tutorías.

\begin{tabular}{cc}
\hline Alfa de Cronbach & $\mathbf{N}^{\circ}$ de elementos \\
\hline, 541 & 2 \\
\hline
\end{tabular}

Fuente: Elaboración propia.

El impacto de la ISO, es el resultado de una encuesta estudiantil de la población, en base a un muestreo piloto de 50 estudiantes, la misma que permitió determinar la probabilidad de ocurrencia $P$ y la probabilidad de no ocurrencia $Q$, siendo los porcentajes de 83 y 17 respectivamente, el resultado arrojo que hay que realizar 200 encuestas , para determinar el impacto de la ISO en la Facultad de Ingeniería de la Universidad Nacional de Chimborazo. La muestra es eminentemente aleatoria, es decir cualquier estudiante puede ser seleccionado para llenar la encuesta. En el tabla 11 se puede observar las variables que intervienen en la muestra.

Tabla 11. Variables para la muestra.

\begin{tabular}{ccl}
\hline No $^{0}$ & Variables & \multicolumn{1}{c}{ Definición } \\
\hline 1 & Z & Nivel de confianza del 95\% \\
2 & E & Margen de error del 5\% \\
3 & P & Probabilidad de ocurrencia 93\% \\
4 & Q & Probabilidad de no ocurrencia 17\% \\
5 & N & Población de estudiantes 2240 \\
\hline
\end{tabular}

Fuente: Elaboración propia.

\section{RESULTADOS}

En la matriz (Tabla 12- Figura 2) se encuentra los estadísticos, los mismos que son analizados en su conjunto. El impacto de la ISO es bueno para todas las preguntas, el número que más se repite en estas preguntas es el 4 (moda). El promedio de la mediana está en 4 , esto significa que el $50 \%$ de los datos está por sobre el 4 y el otro $50 \%$ está por debajo de ese valor. En la Tabla 13 se puede observar el promedio de la desviación, la misma es de 0,77 , pues los datos disponen de cierta variabilidad, puesto que hay calificaciones de 1 y 5 . El promedio general está en 3,67 siendo cercano a bueno.

Los datos de intervalo se transforman a categorías, en la matriz (Tabla 14, Figura 3) se observa que el $0,5 \%$ manifiestan que el impacto de la ISO es malo, el $7 \%$ que es regular, el $80,5 \%$ que es bueno y tan solo el $12 \%$ se inclinan por muy buena.

En la Tabla 15 y Figura 4, se puede observar las dimensiones que pertenecen al modelo de encuestas, la misma refleja que para todas las dimensiones el impacto es bueno, pues el número que más se repite es el 4 (moda). La Figura 3, ratifica lo antes mencionado con una diferencia, que para obtener un impacto muy bueno de la ISO sobre la gestión de calidad la misma debería alcanzar el número 5 en todas sus dimensiones, con lo que se concluye que hay realizar una mejora continua, la mejora continua es una estrategia de la calidad, es un concepto que pretende mejorar los productos, servicios y procesos. Deming (1990). 
Tabla 12. Estadistica descriptiva.

\begin{tabular}{|c|c|c|c|c|c|c|c|c|c|c|c|c|c|c|c|c|c|c|c|c|c|c|c|c|c|}
\hline & & 1 & 2 & 3 & 4 & 5 & 6 & 7 & 8 & 9 & 10 & 11 & 12 & 13 & 14 & 15 & 16 & 17 & 18 & 19 & 20 & 21 & 22 & 23 & 24 \\
\hline & Válidos & 200 & 200 & 200 & 200 & 200 & 200 & 200 & 200 & 200 & 200 & 200 & 200 & 200 & 200 & 200 & 199 & 200 & 200 & 200 & 200 & 200 & 200 & 200 & 200 \\
\hline & Perdidos & 0 & 0 & 0 & 0 & 0 & 0 & 0 & 0 & 0 & 0 & 0 & 0 & 0 & 0 & 0 & 9 & 0 & 0 & 0 & 0 & 0 & 0 & 0 & 0 \\
\hline \multicolumn{2}{|c|}{ Media } & 3,95 & 3,74 & 3,82 & 3,44 & 3,80 & 3,78 & 3,77 & 3,80 & 3,51 & 3,84 & 3,50 & 3,51 & 3,52 & 3,56 & 3,59 & 3,62 & 3,47 & 3,82 & 3,73 & 3,49 & 3,68 & 3,86 & 3,58 & 3,77 \\
\hline \multicolumn{2}{|c|}{ Mediana } & 4,00 & 4,00 & 4,00 & 4,00 & 4,00 & 4,00 & 4,00 & 4,00 & 4,00 & 4,00 & 4,00 & 4,00 & 4,00 & 4,00 & 4,00 & 4,00 & 4,00 & 4,00 & 4,00 & 4,00 & 4,00 & 4,00 & 4,00 & 4,00 \\
\hline \multicolumn{2}{|c|}{ Moda } & 4,00 & 4,00 & 4,00 & 4,00 & 4,00 & 4,00 & 4,00 & 4,00 & 4,00 & 4,00 & 4,00 & 4,00 & 4,00 & 4,00 & 4,00 & 4,00 & 4,00 & 4,00 & 4,00 & 4,00 & 4,00 & 4,00 & 4,00 & 4,00 \\
\hline \multicolumn{2}{|c|}{ Desv. típ. } &, 56 & ,61 & ,68 & ,86 & ,70 & ,70 & 1,55 & ,64 & ,80 & ,66 & ,85 & ,85 & 89 & ,76 &, 77 & ,82 & ,86 & ,68 &, 74 & ,80 & ,74 &, 74 & ,72 & ,69 \\
\hline \multicolumn{2}{|c|}{ Mínimo } & 1,00 & 1,00 & 1,00 & 1,00 & 1,00 & 2,00 & 1,00 & 2,00 & 1,00 & 1,00 & 1,00 & 1,00 & 1,00 & 1,00 & 1,00 & 1,00 & 1,00 & 1,00 & 1,00 & 1,00 & 1,00 & 1,00 & 1,00 & 1,00 \\
\hline \multicolumn{2}{|c|}{ Máximo } & 5,00 & 5,00 & 5,00 & 5,00 & 5,00 & 5,002 & 23,00 & 5,00 & 5,00 & 5,00 & 5,00 & 5,00 & 5,00 & 5,00 & 5,00 & 5,00 & 5,00 & 5,00 & 5,00 & 5,00 & 5,00 & 5,00 & 5,00 & 5,00 \\
\hline
\end{tabular}

Fuente. Elaboración propia.

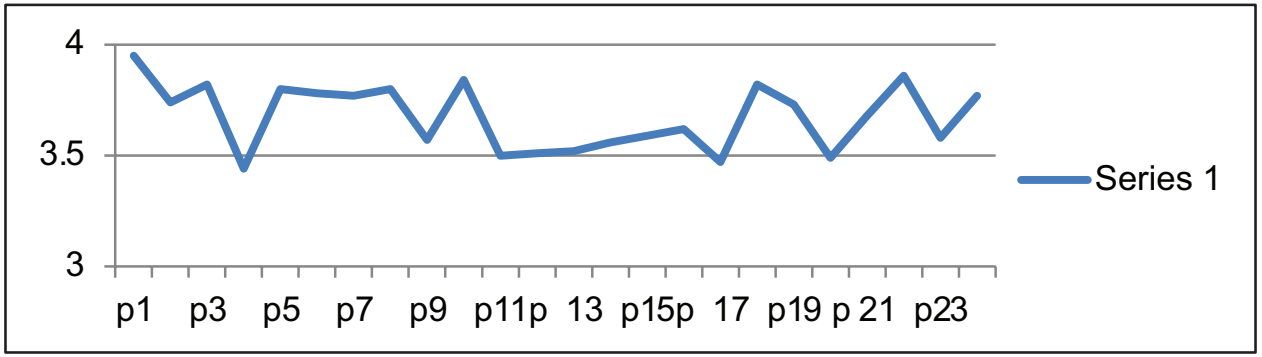

Fuente: Elaboración propia.

Figura 2. Promedio de los datos de la encuesta.

Tabla 13. Promedio de medias y desviación estándar.

\begin{tabular}{rrr}
\hline Preguntas & \multicolumn{1}{c}{ Medias } & Desviación \\
\hline p1 & 3,95 & 0,56 \\
p2 & 3,74 & 0,61 \\
p3 & 3,82 & 0,68 \\
p4 & 3,44 & 0,86 \\
p5 & 3,8 & 0,7 \\
p6 & 3,78 & 0,7 \\
p7 & 3,77 & 1,55 \\
p8 & 3,8 & 0,64 \\
p9 & 3,57 & 0,8 \\
p10 & 3,84 & 0,66 \\
p11 & 3,5 & 0,85 \\
p12 & 3,51 & 0,85 \\
p13 & 3,52 & 0,89 \\
p14 & 3,56 & 0,76 \\
p15 & 3,59 & 0,77 \\
p16 & 3,62 & 0,82 \\
p17 & 3,47 & 0,86 \\
p18 & 3,82 & 0,68 \\
p19 & 3,73 & 0,74 \\
p20 & 3,49 & 0,8 \\
p21 & 3,68 & 0,74 \\
p22 & 3,86 & 0,74 \\
p23 & 3,58 & 0,72 \\
p24 & 3,77 & 0,69 \\
\hline Total & 88,21 & 18,67 \\
\hline Promedio & & 0,77791667 \\
\hline & 3,67541667 &
\end{tabular}

Fuente. Elaboración propia.
Tabla 14. ISO Evaluación.

\begin{tabular}{|c|c|c|c|c|c|}
\hline & & 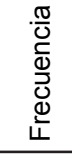 & $\begin{array}{l}\frac{0}{\pi} \\
\stackrel{0}{0} \\
\frac{0}{0} \\
\frac{0}{0} \\
0\end{array}$ & 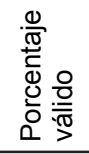 & 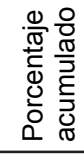 \\
\hline \multirow{5}{*}{ Válidos } & Malo & 1 &, 5 &, 5 &, 5 \\
\hline & Regular & 14 & 7,0 & 7,0 & 7,5 \\
\hline & Bueno & 161 & 80,5 & 80,5 & 88,0 \\
\hline & Muy bueno & 24 & 12,0 & 12,0 & 100,0 \\
\hline & Total & 200 & 100,0 & 100,0 & \\
\hline
\end{tabular}

Fuente. Elaboración propia.

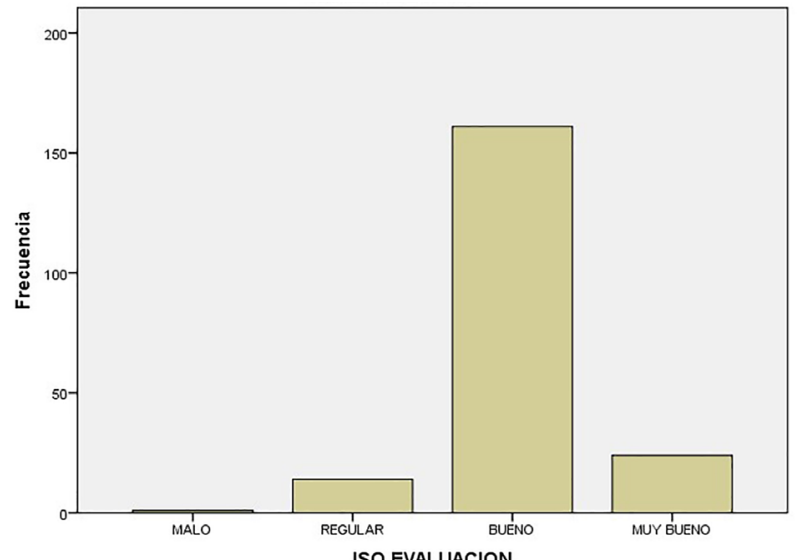

Fuente: Elaboración propia 
Tabla 15. Estadísticos.

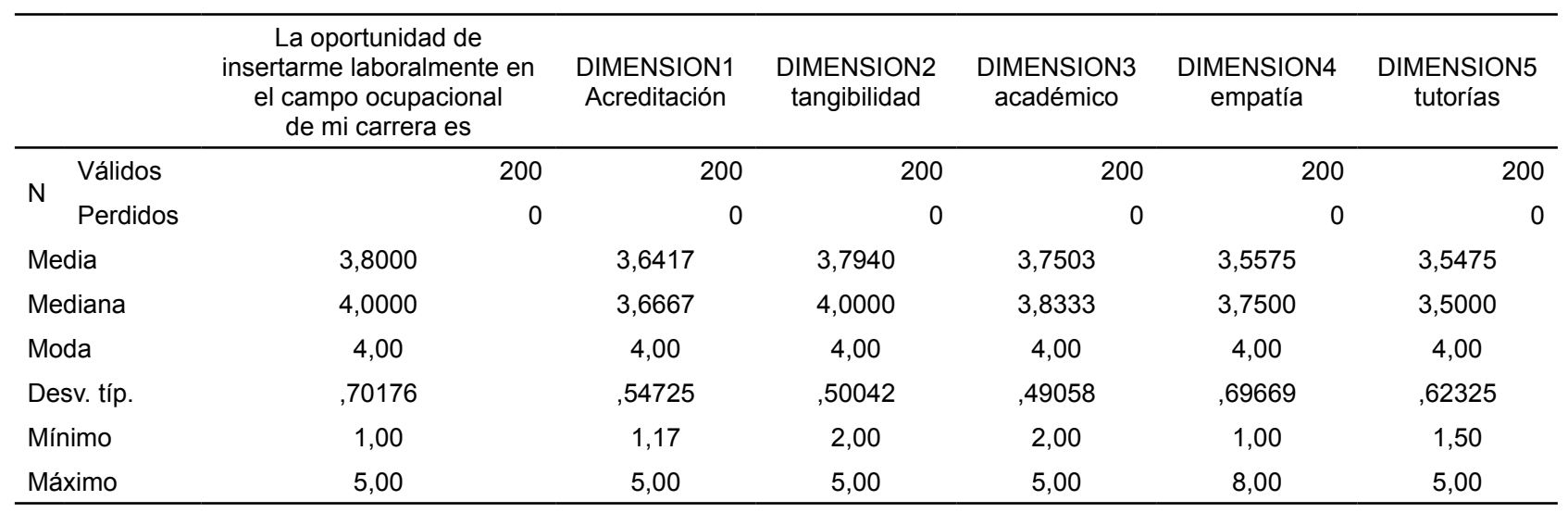

Fuente. Elaboración propia.

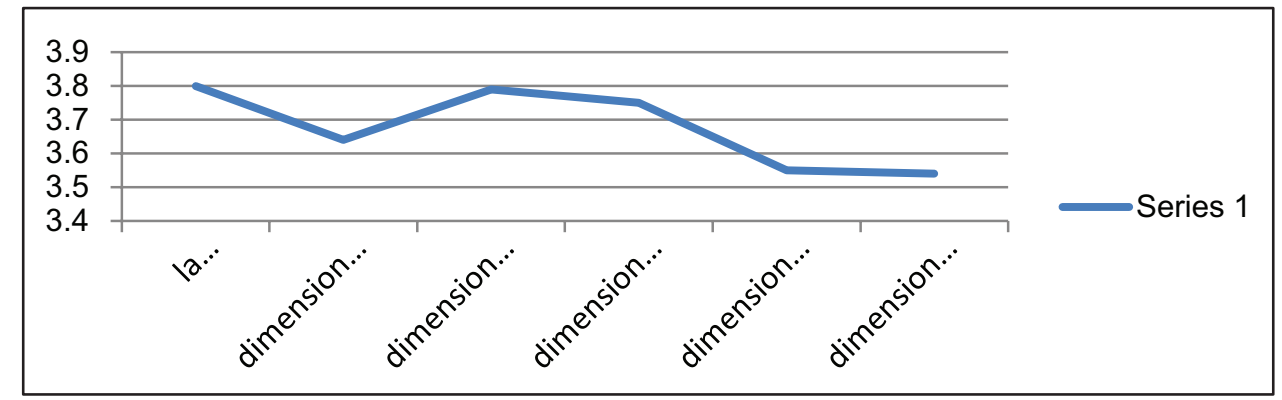

Fuente: Elaboración propia.

Figura 4. Dimensiones del modelo de encuesta.

\section{DISCUSIÓN}

En general los Estudiantes califican de buena el impacto que tiene la ISO 9001:2008 sobre la gestión de calidad, el promedio general es de 3,67 y el número que más se repite en todas las preguntas es el 4 (moda). Para el $0,5 \%$ de los encuestados el impacto es malo sin resultado alguno, para el $7 \%$ el impacto es regular, para el $80,5 \%$ el impacto bueno y solo para el $12 \%$ el impacto es muy bueno.

En la pregunta "La oportunidad de insertarme laboralmente en el campo ocupacional de mi carrera es" El promedio total, arroja una cifra del 3,80\%, lo que representa un $76 \%$ respecto al índice ideal 5 de la escala de Likert. Esto es, que el $76 \%$ de los encuestados consideran que el impacto es bueno según el grado de porcentaje 0-100 puesto que el rango está entre 60-80.

En la dimensión de acreditación, El promedio total, arroja una cifra del 3,64 lo que representa un $72,8 \%$ respecto al índice ideal 5 de la escala de Likert. Esto es, que el $72,8 \%$ de los encuestados consideran que el impacto es bueno según el grado de porcentaje 0-100 puesto que el rango está entre 60-80.

En la dimensión de tangibilidad, El promedio total, arroja una cifra del 3,79 lo que representa un $75,8 \%$ respecto al índice ideal 5 de la escala de Likert. Esto es, que el $75,8 \%$ de los encuestados consideran que el impacto es bueno según el grado de porcentaje 0-100 puesto que el rango está entre 60-80.

En La dimensión académica, El promedio total, arroja una cifra del 3,75 lo que representa un $75 \%$ respecto al índice ideal 5 de la escala de Likert. Esto es, que el $75 \%$ de los encuestados consideran que el impacto es bueno según el grado de porcentaje 0-100 puesto que el rango está entre 60-80.

En La dimensión de empatía, El promedio total, arroja una cifra del 3,55 lo que representa un $71 \%$ respecto al índice ideal 5 de la escala de Likert. Esto es, que el $71 \%$ de los encuestados consideran que el impacto es bueno según el grado de porcentaje $0-100$ puesto que el rango está entre 60-80. 
En La dimensión de tutorías, El promedio total, arroja una cifra del 3,54 lo que representa un $70,8 \%$ respecto al índice ideal 5 de la escala de Likert. Esto es, que el $70,8 \%$ de los encuestados consideran que el impacto es bueno según el grado de porcentaje 0-100 puesto que el rango está entre 60-80.

\section{CONCLUSIÓN}

Esta investigación permitió analizar la percepción que tiene el estudiante con respecto a la ISO 90001:2008 y su impacto sobre la gestión de la calidad, la misma que es buena, pues su promedio es de 3,67. Esta investigación fue de carácter descriptivo con una muestra aleatoria, los datos se recogieron insitu. A través de las cinco dimensiones, utilizando un instrumento apropiado y validado por medio del alfa de crombach.

Al concluir el estudio, se logró cumplir con el propósito establecido, ya que se pudo determinar la situación actual de la Institución respecto a la gestión de la calidad.

\section{RECOMENDACIONES}

Se debe realizar mediciones de la gestión de calidad continuamente, con miras a determinar las necesidades de los clientes.

Se debe elaborar un plan de trabajo, orientado al estudiante y llegar a satisfacer sus necesidades.
La Institución debe enfocarse en mejorar las percepciones en todas sus dimensiones, ya que las mismas generaron porcentajes inferiores al $80 \%$.

En el diseño se debe incrementar preguntas orientadas a los requisitos de la norma ISO, como por ejemplo:

- Requisitos generales.

- Requisitos de la documentación.

- Política de calidad y sus objetivos.

- Manual de calidad, procesos y procedimientos documentados, entre otros.

\section{REFERENCIAS BIBLIOGRÁFICAS}

[1] Dominguez, H. (2006). El servicio invisible, fundamento de un buen servicio al cliente .2a. Ed.,Edit.Ecoe,colombia

[2] Ishikawa, K. (1997). ¿Que es el control de calidad? .1a.Ed., Edit.Norma, Barcelona

[3] Juran, J., \& gryna, F. (1993). Manual de control de calidad .2a.Ed ., Edit.Mc Graw-Hill/Interamericana, Barcelona.

[4] Wellintong, P. (1997). Como brindar un servicio integral de atencion al cliente .1a.Ed ., Edit. Mc Graw-Hillinteramericana, España.

[5] Yanéz, C. (2008). Sistema de gestion de calidad en base a la norma ISO 9001. Internacional evento 